\title{
West Nile virus - Mosquitoes no longer just an annoyance!
}

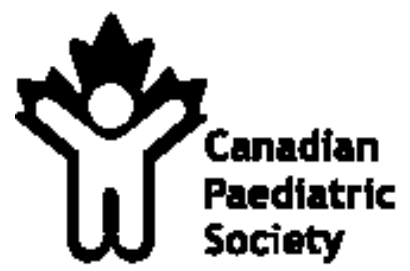

\section{THE EPIDEMIC}

West Nile virus (WNV) was first discovered in Uganda in 1937 and subsequently has been found in several areas in Africa, Asia, the Middle East and Oceania (1-3). More recently, it has appeared in the temperate climates of Europe and has caused large outbreaks in Romania, Russia and Israel (4-6). In 1999, it was first reported in the western hemisphere, when it caused an outbreak of human disease in New York (7). WNV arrived in Canada in the summer of 2001 (8).

WNV is an arbovirus of the Flavivirus genus in the serocomplex that includes Japanese B encephalitis, St Louis encephalitis, and Murray Valley and Kunjin viruses. Culex mosquitoes are the main vectors. Birds are the definitive hosts or reservoirs. WNV is maintained and amplified in a mosquito-bird-mosquito cycle. Birds of the Corvidae family, which includes crows, jays, ravens and magpies, are the major reservoirs in North America. Humans, horses and other vertebrates are incidental hosts, infected by biting mosquitoes, but are not normally sources of transmission because viremia is transient and of low level (1-3). In recent outbreaks, it appears that more severe human disease and higher rates of bird mortality are occurring, suggesting the emergence of more virulent strains (3).

In the initial New York outbreak, there were 62 cases of encephalitis and seven deaths (7). A serosurvey indicated that $2.6 \%$ of the population at the outbreak epicenter in Queens were infected, the majority without symptoms (9). WNV spread to larger areas of the United States in each subsequent summer and by 2002 was found in most states (10). Outbreaks occur in urban areas along waterways where dense bird populations and dense human populations coincide (1).

Infected birds were found in Ontario in the summer of 2001. In August 2002, the first human cases of WNV infection in Canada were reported in an outbreak in Ontario, which resulted in 307 confirmed and 83 probable cases, with 17 deaths. There were 16 confirmed and one probable case in Quebec, with one death. Two cases in Alberta were thought to have been related to travel and not actually acquired there. WNV has been found in birds in Ontario, Quebec, Manitoba, Saskatchewan and Nova Scotia; in horses in Quebec, Ontario, Manitoba and Saskatchewan; and in mosquitoes in Quebec, Ontario and Manitoba (11). Given the wide area of WNV activity, more widespread human disease is expected in Canada in the summer of 2003 when the over-wintering mosquitoes become active.
In temperate climates, WNV activity lasts from May until the end of October, with a peak in late August to early September coinciding with peak mosquito activity. In warmer climates, transmission may occur year-round (1).

Children appear to be at lower risk of serious WNV infection than adults. In endemic countries, WNV infection is mainly a disease of young children, who develop a mild nonspecific febrile illness. Adults are usually immune (1). In countries where the virus has been introduced more recently, older adults have been most severely affected $(4,7,10)$. Surveillance has concentrated on the more severe cases admitted with neurological symptoms, and probably underestimates disease in children. Nevertheless, children may have serious disease. Of the 2354 cases of meningoencephalitis reported in the United States in 2002, the median age was 59 years with a range of one month to 99 years. There were 31 cases $(1.3 \%)$ aged zero to nine years and 58 $(2.5 \%)$ aged 10 to 19 years (10). In Israel, $2.1 \%$ of hospitalized cases were less than 10 years old and $6.9 \%$ were between 10 and 20 years old (6).

Although humans are incidental hosts, human-tohuman transmission has occurred through organ transplantation and by transfusion of blood from infected donors. WNV infection occurred in four recipients of organs from a single infected donor (12). There have been six confirmed cases of transfusion-acquired WNV infection in the United States (13) and one probable case in Canada (14). Intrauterine transmission occurred from a mother with WNV infection at 27 weeks of gestation. Her infant was delivered at term with chorioretinitis and central nervous system dysplasia (15). In another report, an infant of a mother who developed WNV after delivery appeared to have acquired WNV from breast milk. The infant seroconverted but remained asymptomatic (16).

\section{CLINICAL PRESENTATION}

A serosurvey indicated that $80 \%$ of WNV infections were asymptomatic (9). The incubation period for symptomatic disease is three to 14 days after the mosquito bite. A nonspecific febrile illness, West Nile fever, occurs in approximately $20 \%$ of those infected. This illness is usually of abrupt onset with fever, headache, backache, myalgia, malaise and fatigue. Arthralgias, gastrointestinal symptoms, maculopapular rash, ocular pain, pharyngitis, conjunctivitis, lymphadenopathy and respiratory symptoms are less common features. The duration of illness is usually three to

Montreal Children's Hospital, Montreal, Quebec

Correspondence: Dr Dorothy Moore, Montreal Children's Hospital, 2300 Tupper Street, Montreal, Quebec. Telephone 514-412-4485,

fax 514-412-4494, e-mail dorothy.moore@muhc.mcgill.ca 
six days $(1-3,6)$. Information on the clinical presentation of uncomplicated West Nile fever comes from outbreaks in Europe and Israel because North American data published to date has been from patients admitted with severe disease.

Severe infection with encephalitis, meningoencephalitis or meningitis occurs in one in 150 to one in 200 infections. High fever is accompanied by headache, disorientation, confusion, stupor, coma and neck stiffness $(1-2,4-7)$. Encephalitis is more common than meningitis, accounting for approximately $60 \%$ of neurological disease $(6,7)$. Severe muscle weakness and paresis are common, and flaccid paralysis with axonal neuropathy occurred in $10 \%$ of the New York cases (7). This feature may serve to distinguish WNV encephalitis from other etiologies. Rhombencephalitis, cranial nerve abnormalities, myelitis, optic neuritis, polyradiculitis and seizures are less common. Myocarditis, pancreatitis and hepatitis are rare non-neurological complications (1-3). Although children are more likely to present with West Nile fever than with neurological disease, nevertheless, the spectrum of disease reported in children appears to be similar to that in adults $(2,3,7,10)$

The case fatality rate in hospitalized patients in recent outbreaks has been $4 \%$ to $14 \%(4,6,7,10)$. Advanced age, diabetes mellitus and immunosuppression are risk factors for severe disease and death (2,5-7). Long term sequelae of neurological infection include muscle weakness, fatigue, cognitive dysfunction, loss of memory and depression $(1,2)$.

Complete blood counts are usually normal although lymphocytopenia and anemia may occur. In neurological disease, cerebrospinal fluid (CSF) shows mild pleocytosis with lymphocyte predominance, elevated protein and normal glucose. Computed tomography brain scans do not show any acute disease. Magnetic resonance imaging may show enhancement of the meninges or periventricular areas $(1,2,6,7)$.

Treatment is supportive. There is no effective antiviral agent known at present, but new agents are being studied $(1,2)$.

\section{DIAGNOSIS}

Serology is the basis of laboratory diagnosis at present. Tests include haemagglutination inhibition (HI), enzyme immunoassay (ELISA) and plaque reduction neutralization test (PRNT).

Specific WNV immunoglobulin (Ig) M antibody may be detected in serum or CSF by antigen-capture ELISA within eight days of onset. A four-fold or greater increase in IgG antibody titres when acute serum and convalescent serum obtained 21 days after onset are compared indicates seroconversion. HI and ELISA are used as screening tests, but as cross-reactions with other flaviviruses occur, positive results are confirmed by PRNT. Cross-reactions may occur in persons recently vaccinated against yellow fever or Japanese B encephalitis, or infected with dengue or St Louis encephalitis virus. WNV IgM may remain positive for several months, making this test less useful in endemic situations (1-3).

Demonstration of WNV in blood, CSF, tissue or other body fluids by virus isolation or by detection of WNV antigen or genomic sequences is diagnostic but not very sensitive because viremia may be transient and of low level. Sensitivity of TaqMan RT-PCR was reported as $14 \%$ and $57 \%$ in serum and CSF, respectively (17).

\section{SURVEILLANCE}

Suspected cases should be reported promptly because public health interventions may be needed. For surveillance purposes, Health Canada defines a 'suspect' case of WNV meningoencephalitis as an acute febrile illness with clinical findings of encephalitis, meningoencephalitis, meningitis or acute flaccid paralysis, no other etiology found, history of exposure in an area where WNV activity is occurring, and specific WNV diagnostic tests have not been done or results are pending. A 'probable' case is one with this clinical presentation and seroconversion by HI or ELISA, IgM antibody in serum or CSF, a single high IgG antibody titre by HI or ELISA confirmed by PRNT, or having received donor blood that was positive on nucleic acid amplification test. A 'confirmed' case is one with seroconversion by PRNT; seroconversion by HI or ELISA with convalescent serum positive by PRNT; IgM antibody in serum or CSF confirmed by a positive PRNT; or documentation of virus, virus antigen or genomic sequence in blood, CSF or tissue. Clinical criteria for West Nile fever are fever and at least one of myalgia, arthalgia, headache, fatigue, photophobia, lymphadenopathy or maculopapular rash, with a history of exposure as above. Laboratory criteria are as for WNV meningoencephalitis. Surveillance definitions may vary slightly in different provinces. Active surveillance of WNV activity in birds, horses and mosquitoes is also carried out in most provinces (11). Outbreaks are usually detected in birds before cases occur in humans (10).

\section{PREVENTION}

Although studies are underway, there is no vaccine currently available $(1,2)$. Prevention is by protection from the bite of an infected mosquito. In areas of WNV transmission, protection of children should be considered during outdoor activities, including those related to school, day care or camp. Education of families and other caretakers is an important component of prevention (18). Specific measures include:

\section{Reduction of mosquito population:}

Mosquitoes breed in standing water. Measures to limit mosquito breeding around the home include drainage or removal of receptacles for standing water (old tires, toys, flower pots, cans, buckets, barrels and other containers that may collect rain water); keeping swimming pools, decorative pools, children's wading pools, bird-baths, etc, clean; and cleaning clogged gutters. Under certain circumstances, mosquito control measures may be carried out by public health, such as drainage of standing water sources, use of microbiological larvicides in waters that are mosquito breeding grounds, and surface spraying to control adult vectors $(1,2,18)$.

\section{Reduction of exposure to mosquitoes:}

Avoiding infested areas, limiting outdoor activities at times of high mosquito activity such as dawn and dusk, and screening of windows and doors reduce exposure. Mosquito traps and ultrasonic and other devices designed to distract mosquitoes from people are not well studied but appear to have limited activity and may in fact attract more mosquitoes into the area $(1,2,18,19)$. 
3. Use of barriers to protect skin:

Barriers include mosquito nets, screens for baby strollers or other areas where immobile children are placed, protective light-coloured clothing with long cuffed sleeves, long pants tucked into socks or shoes, and hats $(1,2,18)$.

\section{Discourage mosquitoes from biting:}

Insect repellents do not kill mosquitoes but act by making the human unattractive to the mosquito. Repellents should be used if outdoors when mosquitoes are around, especially in areas of WNV transmission (1,2,19-22). Repellents are synthetic compounds or derivatives of plant oils. Of the products currently available, only N,Ndiethyl-3-methylbenzamide (DEET) based products provide adequate long-lasting protection during sustained outdoor activity. More concentrated DEET products protect longer, and are appropriate for persons who will be exposed to mosquitoes during outdoor activities lasting many hours. Lower concentrations may be used when more transient protection is required but may require repeated applications. A study in human volunteers showed average durations of protection of $5 \mathrm{~h}, 4 \mathrm{~h}, 2 \mathrm{~h}$ and $1.5 \mathrm{~h}$ for DEET concentrations of $23.8 \%, 20 \%, 6.65 \%$ and $4.54 \%$, respectively. A $2 \%$ soybean oil based product protected for $1.5 \mathrm{~h}$, and in a preliminary study, oil of eucalyptus appeared to be protective for $2 \mathrm{~h}$. All other plant products studied, including those based on citronella, protected for less than $20 \mathrm{~min}$. Ingestion of garlic or vitamin $\mathrm{B} 1$, wearing devices that emit sounds or using impregnated wristbands are all ineffective measures $(19,22)$. DEET has been used worldwide since 1957, has been studied more extensively than any other repellent and has a good safety profile $(19,20,22)$. DEET is used by about $21 \%$ of households, $30 \%$ of adults and $34 \%$ of children (21). Nevertheless, there is considerable concern about potential toxicity, especially in children. Adverse effects are rare and most often associated with chronic or excessive use, and do not appear to be related to the DEET concentration used. Urticaria and contact dermatitis have been reported in a small number of people. There have been rare reports of encephalopathy, with 13 cases reported after skin application in children (19). Encephalopathy has also been reported after accidental ingestion (23). DEET is irritating to the eyes and mucous membranes. Concentrated formulations may damage plastic and certain fabrics (19). If used appropriately, DEET does not present a health problem and in areas of WNV activity the risk of infection must be balanced against concerns about toxicity.

Although concentrations of less than 10-15\% DEET have been recommended for children, there is no evidence that these concentrations are safer than 30\% DEET. There is also no evidence that non-DEET repellents are safer and there is no safety data on other products in children. In 2002, the Canadian Pest Management Regulatory Agency recommended that:

DEET should not be used on children younger than six months of age; children aged six months to two years should be limited to one application per day; children aged two to 12 years should be limited to three applications per day; and the maximum concentration used should be $10 \%$ or less for children up to 12 years of age and up to $30 \%$ for those over 12 years (24).

These recommendations are based on theoretical calculations of potential absorption and toxicity using animal models. On the other hand, the United States Environmental Protection Agency in 2001 concluded that normal use of DEET at concentrations of up to $30 \%$ posed no significant risk to children or adults, but that DEET should not be used in children younger than two months of age because of increased skin permeability. The American Academy of Pediatrics has supported the agency's recommendation (21).

Recommendations for use of DEET in children $(21,25,26)$ are as follows:

- Do not apply over cuts, wounds, or irritated or sunburned skin. Avoid areas around eyes and mouth.

- Do not spray onto the face - apply with hands.

- Use just enough to cover exposed skin. Avoid using excessive amounts or using excessive numbers of applications.

- Do not apply to a young child's hands (they may rub it into their eyes or mouth).

- Do not allow young children to apply the product themselves.

- Do not apply under clothing.

- Do not use sprays in enclosed areas or near food.

- Avoid preparations of DEET combined with sunscreen because sunscreen may need to be applied more often.

- Reapply if washed off by sweating or by getting wet.

- After returning indoors, wash off with soap and water.

Treating clothes with repellents such as DEET or permethrin will give added protection because mosquitoes may bite through thin clothing. Permethrin should not be used on the skin. Mosquito netting may also be impregnated with repellents. Repellents should not be used on clothing or mosquito nets that young children may chew or suck on.

Other measures include prevention of WNV infection by blood or organ donation. Donors should be screened for recent febrile illness. A sensitive test for WNV nucleic acid that could be used to screen donors is being developed. Patients with WNV infection should be asked about recent blood donation. Public health should be notified if a patient develops WNV within two weeks of blood or organ donation and likewise if WNV infection develops within four weeks of receiving a transfusion or a transplant so that any remaining blood products from suspect donors may be withdrawn from circulation. Pregnant women should be advised to take measures to prevent mosquito bites. There is no recommendation for an infected mother to avoid breastfeeding because the only case of transmission via breast milk reported to date was asymptomatic.

\section{KEY POINTS}

- WNV is present in Canada and is expected to become more widespread in the summer of 2003. 
- Serious disease is more common in adults and most children are expected to have mild disease, but severe disease can occur in children. Little is known of the spectrum of disease in children in the North American epidemic.

- Immunocompromised children may be at risk for more severe disease. Counselling about camp or other outdoor activities should take into consideration the ability of the child to comply with preventive measures, the degree to which the child is immunocompromised, the likelihood of WNV transmission in the area and the extent of exposure to mosquitoes.

- WNV should be considered in the differential diagnoses of children presenting with acute encephalitis, meningoencephalitis or meningitis during the season of mosquito activity, especially if enteroviral infection has been excluded.

- Suspected cases should be reported promptly to public health.

- There is no antiviral treatment at present.

- There is no vaccine at present. Prevention is by avoidance of mosquito bites. Effective mosquito repellents can be used in children and are safe if used appropriately.

- Parents should be informed about the possibility of WNV activity in Canada and measures they may take to reduce the risk of their children becoming infected.

Further information is available from Health Canada at: http://www.hc-sc.gc.ca/pphb-dgspsp/wnv-vwn/index.html and http://www.hcsc.gc.ca/pphb-dgspsp/publicat/info/, and from the Centers for Disease Control in the United States at http://www.cdc.gov/ncidod/dvbid/westnile

\section{REFERENCES}

1. Campbell GL, Marfin AA, Lanciotti RS, Gubler DJ. West Nile virus. Lancet Infect Dis 2002;2:519-29.

2. Petersen LR, Marfin AA. West Nile virus: A primer for the clinician. Ann Intern Med 2002;137:173-9.

3. Meek J. West Nile virus in the United States. Curr Opin Pediatr 2002;14:72-7.

4. Tsai TF, Popovici F, Cernescu C, Campbell GL, Nedelcu NI. West Nile encephalitis epidemic in southeastern Romania. Lancet 1998;352:767-71.

5. Platonov AE, Shipulin GA, Shipulina OY, et al. Outbreak of West Nile virus infection, Volgograd Region, Russia, 1999. Emerg Infect Dis 2001;7:128-32.
6. Chowers MY, Lang R, Nassar F, et al. Clinical characteristics of the West Nile fever outbreak, Israel, 2000. Emerg Infect Dis 2001;7:675-8.

7. Nash D, Mostashari F, Fine A, et al. The outbreak of West Nile virus infection in the New York City area in 1999. N Engl J Med 2001;344:1807-14.

8. Health Canada, Population and Public Health Branch. West Nile (WN) virus: Canada. Infectious Diseases News Brief August 23, 2001. $<$ http://www.hc-sc.gc.ca/pphb-dgspsp/bid-bmi/dsd-dsm/nbab/2002/nb3402_e.html> (Version current at May 16, 2003).

9. Mostashari F, Bunning ML, Kitsutani PT, et al. Epidemic West Nile encephalitis, New York, 1999: Results of a household-based seroepidemiological survey. Lancet 2001;358:261-4.

10. Centers for Disease Control and Prevention. Provisional surveillance summary of the West Nile virus epidemic-United States, JanuaryNovember 2002. Morb Mortal Wkly Rep 2002;51:1129-33.

11. Health Canada, Population and Public Health Branch. West Nile virus. Surveillance data. April 9, 2003. <http://www.hc-sc.gc.ca/pphbdgspsp/wnv-vwn/index.html> (Version current at May 16, 2003).

12. Centers for Disease Control and Prevention. Update: Investigations of West Nile virus infections in recipients of organ transplantation and blood transfusion. Morb Mortal Wkly Rep 2002;51:833-36

13. Centers for Disease Control and Prevention. Investigations of West Nile virus infections in recipients of blood transfusion. Morb Mortal Wkly Rep 2002;51:973-4.

14. Sibbald B. Canada will check donor blood for West Nile virus if test available. CMAJ 2003;168:207.

15. Centers for Disease Control and Prevention. Intrauterine West Nile virus infection - New York, 2002. Morb Mortal Wkly Rep 2002;51:1135-6.

16. Centers for Disease Control and Prevention. Possible West Nile virus transmission to an infant through breast-feeding-Michigan, 2002. Morb Mortal Wkly Rep 2002;51:877-8.

17. Lanciotti RS, Kerst AJ, Nasci RS, et al. Rapid detection of West Nile virus from human clinical specimens, field-collected mosquitoes, and avian samples by a TaqMan reverse transcriptase-PCR assay. J Clin Microbiol 2000;38:4066-71.

18. Harrison TW. West Nile encephalitis. J Pediatr Health Care 2002;16:278-81.

19. Fradin MS. Mosquitoes and mosquito repellents: A clinician's guide. Ann Intern Med 1998;128:931-40.

20. Pollack RJ, Kiszewski AE, Spielman A. Repelling mosquitoes. N Engl J Med 2002;347:2-3.

21. Weil WB. New information leads to changes in DEET recommendations. AAP News August 2001. < http://www.aap.org/ family/wnv\%2Daug01.htm> (Version current at May 16, 2003)

22. Fradin MS, Day JF. Comparative efficacy of insect repellents against mosquito bites. N Engl J Med 2002;347:13-8.

23. Petrucci N, Sardini S. Severe neurotoxic reaction associated with oral ingestion of low-dose diethyltoluamide-containing insect repellent in a child. Pediatr Emerg Care 2000;16:341-2.

24. Health Canada, Pest Management Regulatory Agency. Personal insect repellents containing DEET (NN-diethyl-m-toluamide and related compounds). Publications Coordinator, Pest Management Regulatory Agency, Ottawa. April 15, 2002. <http://www.hc-sc.gc.ca/pmra-arla/ english/pdf/rrd/rrd2002-01-e.pdf > (Version current at May 16, 2003).

25. Canadian Paediatric Society. Insect repellents for children. July 2002. <www.caringforkids.cps.ca/keepingkidssafe/repellents.htm> (Version current at May 16, 2003).

26. Centers for Disease Control, Division of Vector Borne Infectious Diseases. Insect repellent use and safety. <http://www.cdc.gov/ncidod/dvbid/ westnile/qa/insect_repellent.htm > (Version current at May 16, 2003).

\section{CANADIAN PAEDIATRIC SOCIETY, INFECTIOUS DISEASES AND IMMUNIZATION COMMITTEE}

Committee Members: Drs. Upton Allen, The Hospital for Sick Children, Toronto, Ontario; H Dele Davies, East Lansing, Michigan (USA); Simon Richard Dobson, BC's Children Hospital, Vancouver, British Columbia; Joanne Embree, The University of Manitoba, Winnipeg, Manitoba (Chair); Joanne Langley, IWK Health Centre, Halifax, Nova Scotia; Dorothy Moore, Montreal Children's Hospital, McGill University Health Centre, Montréal, Québec; Gary Pekeles, The Montreal Children's Hospital, Montreal, Quebec (Director Responsible)

Consultants: Dr Noni MacDonald, Dalhousie University, Halifax, Nova Scotia; Dr Gilles Delage, Héma-Québec

Liaisons: Drs Scott Halperin, IWK Health Centre, Halifax, Nova Scotia (IMPACT); Susan King, The Hospital for Sick Children, Toronto, Ontario (Canadian Paediatrics AIDS Research Group); Larry Pickering, Centre for Pediatric Research, Norfolk, Virginia (American Academy of Pediatrics)

Principal author: Dr Dorothy Moore, Montreal Children's Hospital, McGill University Health Centre, Montréal, Quebec

The recommendations in this statement do not indicate an exclusive course of treatment or procedure to be followed. Variations, taking into account individual circumstances, may be appropriate. This article also appears in Paediatr Child Health 2003;8(5):267-270.

Internet addresses are current at time of publication. 


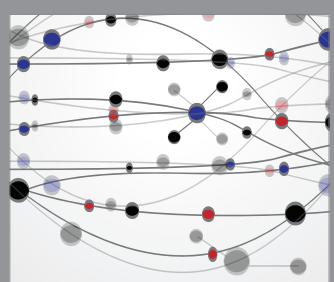

The Scientific World Journal
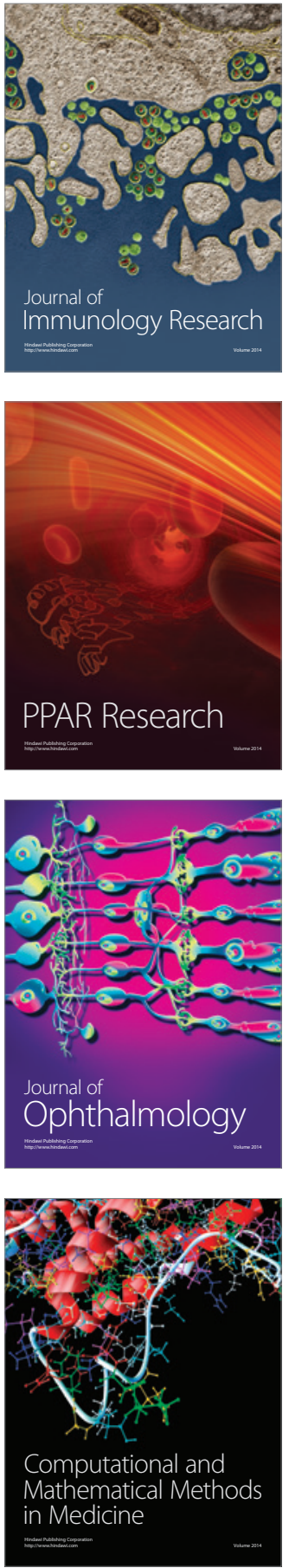

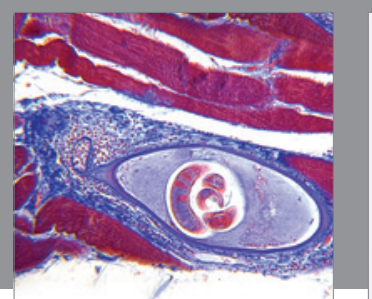

Gastroenterology Research and Practice

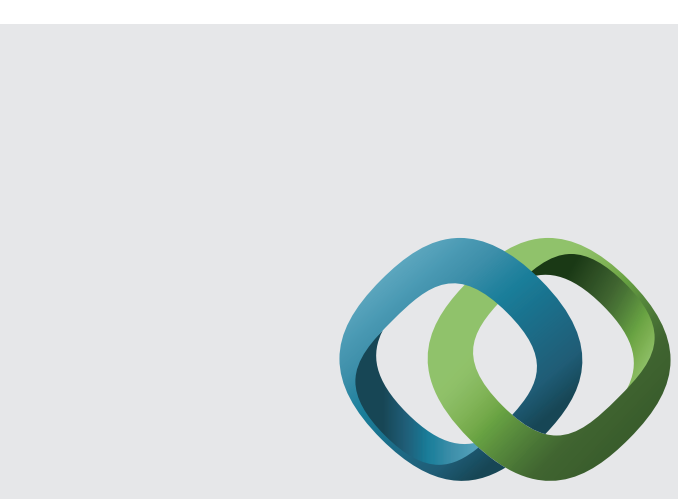

\section{Hindawi}

Submit your manuscripts at

http://www.hindawi.com
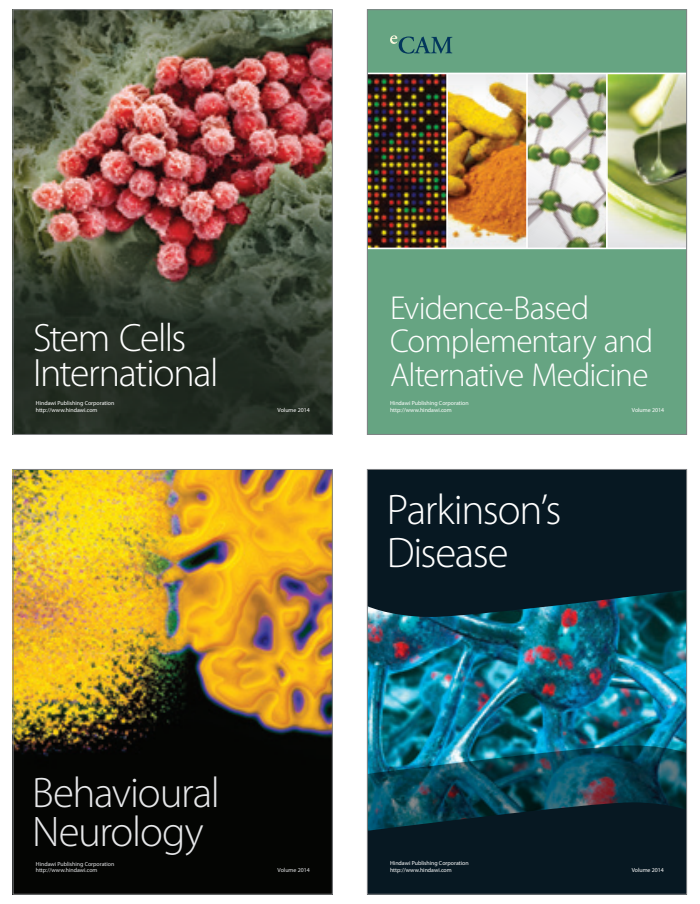
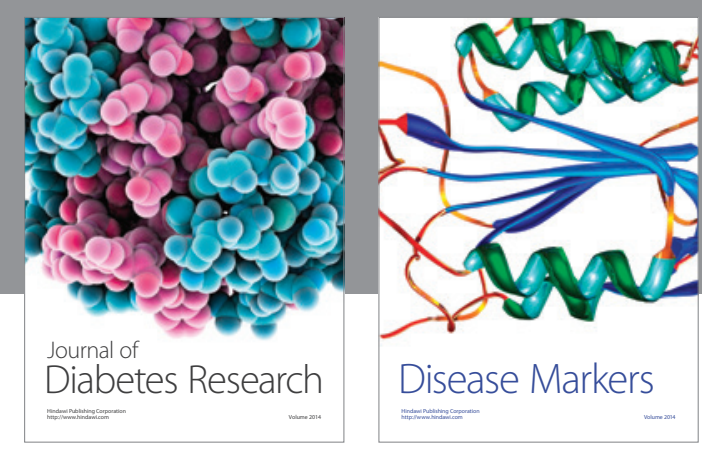

Disease Markers
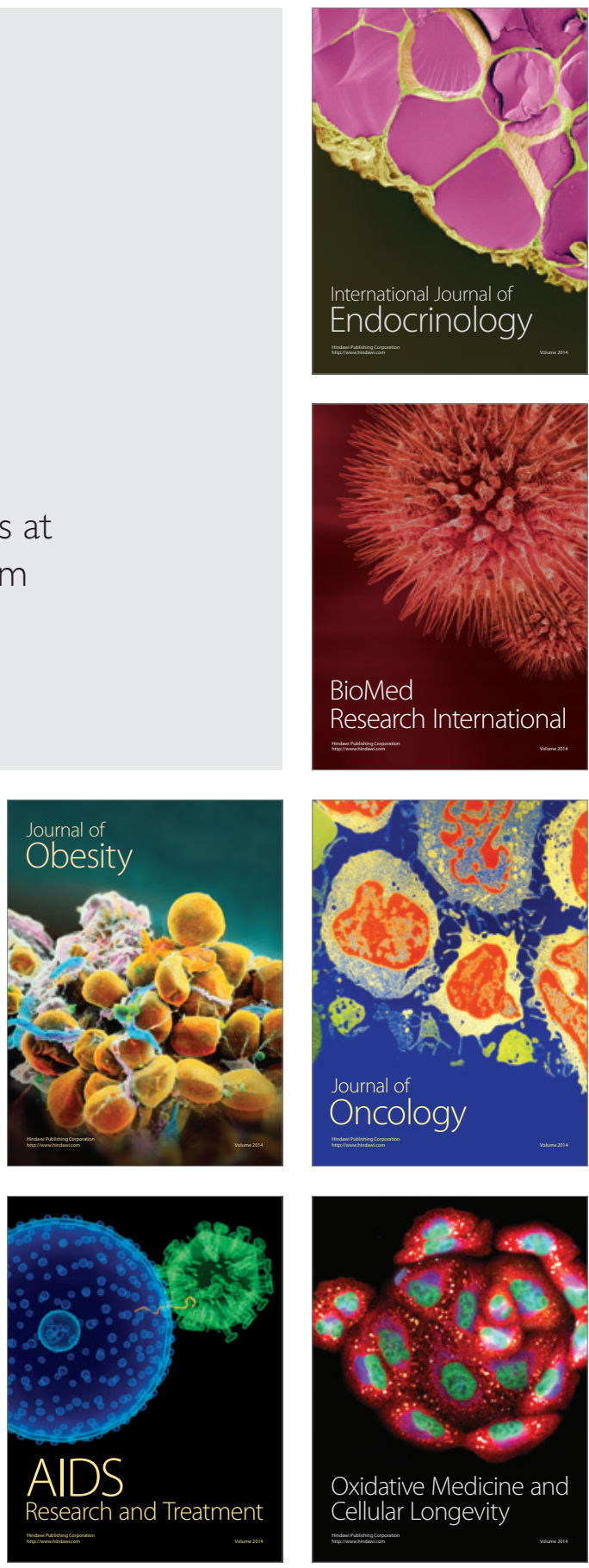